\title{
Convergence of an Iterative Algorithm for Common Solutions for Zeros of Maximal Accretive Operator with Applications
}

\author{
Uamporn Witthayarat, ${ }^{1}$ Yeol Je Cho, ${ }^{2}$ and Poom Kumam ${ }^{1}$ \\ ${ }^{1}$ Department of Mathematics, Faculty of Science, King Mongkut's University of \\ Technology Thonburi (KMUTT), Bangmod, Bangkok 10140, Thailand \\ ${ }^{2}$ Department of Mathematics Education and RINS, Gyeongsang National University, \\ Chinju 660-701, Republic of Korea \\ Correspondence should be addressed to Yeol Je Cho, yjcho@gnu.ac.kr and Poom \\ Kumam, poom.kum@kmutt.ac.th
}

Received 18 November 2011; Accepted 30 December 2011

Academic Editor: Yeong-Cheng Liou

Copyright (C) 2012 Uamporn Witthayarat et al. This is an open access article distributed under the Creative Commons Attribution License, which permits unrestricted use, distribution, and reproduction in any medium, provided the original work is properly cited.

The aim of this paper is to introduce an iterative algorithm for finding a common solution of the sets $\left(A+M_{2}\right)^{-1}(0)$ and $\left(B+M_{1}\right)^{-1}(0)$, where $\mathrm{M}$ is a maximal accretive operator in a Banach space and, by using the proposed algorithm, to establish some strong convergence theorems for common solutions of the two sets above in a uniformly convex and 2-uniformly smooth Banach space. The results obtained in this paper extend and improve the corresponding results of Qin et al. 2011 from Hilbert spaces to Banach spaces and Petrot et al. 2011. Moreover, we also apply our results to some applications for solving convex feasibility problems.

\section{Introduction}

Let $E$ be a real Banach space with norm $\|\cdot\|$ with the dual space $E^{*}$ and let $\langle\cdot, \cdot\rangle$ denote the pairing between $E$ and $E^{*}$. Let $C$ be a nonempty closed convex subset of $E$. We define the generalized duality mapping $J_{q}: E \rightarrow 2^{E^{*}}$ by

$$
J_{q}(x)=\left\{f \in E^{*}:\langle x, f\rangle=\|x\|^{q},\|f\|=\|x\|^{q-1}\right\}, \quad \forall x \in E,
$$

for all $q>1$. In the special case, for $q=2$, we called the mapping $J_{2}$ as the normalized duality mapping and as usual we write $J_{2}=J$. The following is the well-known properties of the generalized duality mapping $J_{q}$ : 
(1) $J_{q}(x)=\|x\|^{q-2} J_{2}(x)$ for all $x \in E$ with $x \neq 0$;

(2) $J_{q}(t x)=t^{q-1} J_{q}(x)$ for all $x \in E$ and $t \in[0, \infty)$;

(3) $J_{q}(-x)=-J_{q}(x)$ for all $x \in E$.

It is well known that if $X$ is smooth, then $J$ is single valued, which is denoted by $j$. Recall that the duality mapping $j$ is said to be weakly sequentially continuous if, for each sequence $\left\{x_{n}\right\}$ with $x_{n} \rightarrow x$ weakly, we have $j\left(x_{n}\right) \rightarrow j(x)$ weakly*. We know that, if $X$ admits a weakly sequentially continuous duality mapping, then $X$ is smooth. For the details, see [1-3].

Let $U=\{x \in E:\|x\|=1\}$. A Banach space $E$ is said to be

(1) uniformly convex if there exists $\delta>0$ such that, for any $x, y \in U$ and, for any $\epsilon \in$ $(0,2],\|x-y\| \geq \epsilon$ implies $\|(x+y) / 2\| \leq 1-\delta$. convex.

We can see that every uniformly convex Banach space is also reflexive and strictly

(2) Smooth if $\lim _{t \rightarrow 0}(\|x+t y\|-\|x\|) / t$ exists for all $x, y \in U$.

(3) Uniformly smooth if the limit is attained uniformly for $x, y \in U$. The modulus of smoothness of $E$ is defined by

$$
\rho(\tau)=\sup \left\{\frac{1}{2}(\|x+y\|+\|x-y\|)-1: x, y \in E,\|x\|=1,\|y\|=\tau\right\},
$$

where $\rho:[0, \infty) \rightarrow[0, \infty)$ is a function. In the other way, $E$ is uniformly smooth if and only if $\lim _{\tau \rightarrow 0} \rho(\tau) / \tau=0$.

(4) q-uniformly smooth if there exists a constant $c>0$ such that $\rho(\tau) \leq c \tau^{q}$ for all $\tau>0$ where $q$ is a fixed real number with $1<q \leq 2$. (see, for instance, $[1,4]$ ).

We note that $E$ is a uniformly smooth Banach space if and only if $J_{q}$ is single valued and uniformly continuous on any bounded subset of $E$. Examples of both uniformly convex and uniformly smooth Banach spaces are $L^{p}$, where $p>1$. More precisely, $L^{p}$ is $\min \{p, 2\}$ uniformly smooth for any $p>1$. Note also that no Banach space is $q$-uniformly smooth for $q>2$ (see $[1,5]$ for more details).

Let $A: C \rightarrow E$ be a nonlinear mapping. The mapping $A$ is said to be

(1) accretive if

$$
\langle A x-A y, J(x-y)\rangle \geq 0, \quad \forall x, y \in C,
$$

(2) $\lambda$-strongly accretive if there exists a constant $\alpha>0$ such that

$$
\langle A x-A y, J(x-y)\rangle \geq \lambda\|x-y\|^{2}, \quad \forall x, y \in C,
$$

(3) $\lambda$-inverse-strongly accretive if there exists a constant $\alpha>0$ such that

$$
\langle A x-A y, J(x-y)\rangle \geq \lambda\|A x-A y\|^{2}, \quad \forall x, y \in C,
$$


Definition 1.1. Let $M: E \rightarrow 2^{E}$ be a multivalued maximal accretive mapping. The singlevalued mapping $J_{M, \rho}: E \rightarrow E$ defined by

$$
J_{M, \rho}(u)=(I+\rho M)^{-1}(u), \quad \forall u \in E,
$$

is called the resolvent operator associated with $M$, where $\rho$ is any positive number and $I$ is the identity mapping.

Let $T$ be a mapping from $E$ into itself. We use $F(T)$ to denote the set of fixed points of the mapping $T$. Recall that the mapping $T$ is said to be nonexpansive if

$$
\|T x-T y\| \leq\|x-y\|, \quad \forall x, y \in E .
$$

A mapping $f: C \rightarrow C$ is said to be contractive if there exists a constant $\alpha \in(0,1)$ such that

$$
\|f(x)-f(y)\| \leq \alpha\|x-y\|, \quad \forall x, y \in C
$$

Recently, Aoyama et al. [4] considered the following generalized variational inequality problem in a smooth Banach space: Find a point $x \in C$ such that

$$
\langle A x, j(y-x)\rangle \geq 0, \quad \forall y \in C,
$$

where $A$ is an accretive operator of $C$ into $E$. This problem is related to the fixed point problem for nonlinear mappings, the problem of finding a zero point of an accretive operator, and so on. For the problem of finding a zero point of an accretive operator by the proximal point algorithm, see Agarwal et al. [6], Cho et al. [7, 8], Kamimura and Takahashi [9, 10], Qin et al. [11], Song et al. [12], and Wei and Cho [13]. In order to find a solution of the variational inequality (1.9), Aoyama et al. [4] studied the weak convergence theorem for accretive operators in Banach spaces, which is a generalization of the result by Iiduka et al. [14] from the class of Hilbert spaces.

Theorem AIT (see [4], Aoyama et al. Theorem 3.1). Let E be a uniformly convex and 2-uniformly smooth Banach space and let $C$ be a nonempty closed convex subset of $E$. Let $Q_{C}$ be a sunny nonexpansive retraction from $E$ onto $C, \alpha>0$ and $A$ be an $\alpha$-inverse strongly accretive operator of $C$ into $E$ with $S(C, A) \neq \emptyset$, where

$$
S(C, A)=\left\{x^{*} \in C:\left\langle A x^{*}, j\left(x-x^{*}\right)\right\rangle \geq 0, \forall x \in C\right\} .
$$

If $\left\{\lambda_{n}\right\}$ and $\left\{\alpha_{n}\right\}$ are chosen such that $\lambda_{n} \in\left[a, \alpha / K^{2}\right]$ for some $a>0$ and $\alpha_{n} \in[b, c]$ for some $b, c$ with $0<b<c<1$, then the sequence $\left\{x_{n}\right\}$ defined by the following manners: $x_{1}=x \in C$ and

$$
x_{n+1}=\alpha_{n} x_{n}+\left(1-\alpha_{n}\right) Q_{C}\left(x_{n}-\lambda_{n} A x_{n}\right), \quad \forall n \geq 1,
$$

converges weakly to an element $z$ of $S(C, A)$, where $K$ is the 2-uniformly smoothness constant of $E$ and $Q_{C}$ is a sunny nonexpansive retraction. 
In 2011, Katchang and Kumam [15] presented an iterative algorithm for finding a common solution of fixed point problems and a general system of variational inequality problems for two accretive operators as shown in the following: for all $n \geq 0$,

$$
\begin{aligned}
x_{0} & =u \in C, \\
y_{n} & =Q_{C}\left(x_{n}-\mu B x_{n}\right), \\
x_{n+1} & =\alpha_{n} f\left(x_{n}\right)+\beta_{n} x_{n}+\gamma_{n} S Q_{C}\left(y_{n}-\lambda A y_{n}\right) .
\end{aligned}
$$

They proved that the sequence $\left\{x_{n}\right\}$ generated by the above algorithm converges strongly to a point $\bar{x}=Q_{\mp} f(\bar{x})$. Moreover, they apply their theorem to find zeros of accretive operators and the class of $k$-strictly pseudocontractive mappings.

Recently, Petrot et al. [16] considered the problem so-called quasivariational inclusion problem, that is, determine an element $u \in H$ such that

$$
0 \in A(u)+M(u)
$$

where $A: H \rightarrow H$ is a single-valued nonlinear mapping and $M: H \rightarrow 2^{H}$ is a multivalued mapping. The set of solutions of the above problem is denoted by $\operatorname{VI}(H, A, M)$. Therefore, they presented a new iterative scheme for finding a common element of the set of fixed points of a nonexpansive mapping and the set of solutions of variational inclusion problem with a multivalued maximal monotone mapping and an $\alpha$-inverse-strongly monotone mapping by using the iterative sequence $\left\{x_{n}\right\}$ defined as follows:

$$
\begin{aligned}
x_{0} & \in H, \text { chosen arbitrarily, } \\
x_{n+1} & =\alpha_{n} f\left(x_{n}\right)+\beta_{n} x_{n}+\gamma_{n} S z_{n}, \\
z_{n} & =J_{M}^{\lambda}\left(y_{n}-\lambda A y_{n}\right), \\
y_{n} & =J_{M}^{\lambda}\left(x_{n}-\lambda A x_{n}\right), \quad \forall n \geq 0,
\end{aligned}
$$

and, under appropriated conditions, they proved the the sequence $\left\{x_{n}\right\}$ generated by (1.14) converges strongly to a point $z_{0} \in H$, which is the unique solution in $F(S) \cap \operatorname{VI}(H, A, M)$ to the following variational inequality:

$$
\left\langle(f-I) z_{0}, z_{0}-z\right\rangle \leq 0, \quad \forall z \in F(S) \cap \operatorname{VI}(H, A, M)
$$

Very recently, Qin et al. [17] introduced an iterative scheme for a general variational inequality (VI) and proved the strong convergence theorems of common solutions of two 
variational inequalities in a uniformly convex and 2-uniformly smooth Banach space by using the following iterative sequence $\left\{x_{n}\right\}$ :

$$
\begin{aligned}
x_{0} & =u \in C, \\
y_{n} & =\delta_{n} Q_{C}\left(x_{n}-\rho B x_{n}\right)+\left(1-\delta_{n}\right) Q_{C}\left(x_{n}-\lambda A x_{n}\right), \\
x_{n+1} & =\alpha_{n} u+\beta_{\mathrm{n}} x_{n}+\gamma_{n} y_{n}, \quad \forall n \geq 0 .
\end{aligned}
$$

They proved that the sequence $\left\{x_{n}\right\}$ generated by the above algorithm converges strongly to a point $q=Q_{\mathrm{VI}} u$, where $Q_{\mathrm{VI}}$ is the unique sunny nonexpansive retraction from $C$ onto VI.

Motivated and inspired by the above recent works, in this paper, we introduce an iterative scheme for finding zeros of maximal accretive operators. Furthermore, we prove some strong convergence theorems and also propose applications for solving the convex feasibility problems. Our results improve and extend the corresponding results of Qin et al. [17] and Katchang and Kumam [15], Petrot et al. [16], and many others.

\section{Preliminaries}

Note that, if $C$ and $D$ are nonempty subsets of a Banach space $E$ such that $D$ is a subset of a closed convex subset $C$ and $Q: C \rightarrow D$. Then $Q$ is said to be sunny if

$$
Q(Q x+t(x-Q x))=Q x
$$

whenever $Q x+t(x-Q x) \in C$ for any $x \in C$ and $t \geq 0$. A subset $D$ of $C$ is said to be a sunny nonexpansive retract of $C$ if there exists a sunny nonexpansive retraction $Q$ of $C$ onto $D$. A mapping $Q: C \rightarrow C$ is called a retraction if $Q^{2}=Q$. If a mapping $Q: C \rightarrow C$ is a retraction, then $Q z=z$ for all $z$ is in the range of $Q$ (see $[4,18]$ for more details).

The following result describes a characterization of sunny nonexpansive retractions on a smooth Banach space.

Proposition 2.1 (see [19]). Let $E$ be a smooth Banach space and let $C$ be a nonempty subset of $E$. Let $Q: E \rightarrow C$ be a retraction and let $J$ be the normalized duality mapping on $E$. Then the following are equivalent:

(1) $Q$ is sunny and nonexpansive;

(2) $\|Q x-Q y\|^{2} \leq\langle x-y, J(Q x-Q y)\rangle$ for all $x, y \in E$;

(3) $\langle x-Q x, J(y-Q x)\rangle \leq 0$ for all $x \in E$ and $y \in C$.

Proposition 2.2 (see [20]). Let $C$ be a nonempty closed convex subset of a uniformly convex and let uniformly smooth Banach space $E$ and $T$ be a nonexpansive mapping of $C$ into itself with $F(T) \neq \emptyset$. Then the set $F(T)$ is a sunny nonexpansive retract of $C$.

We need the following lemmas in order to prove our main results. 
Lemma 2.3 (see [5]). Let E be a real 2-uniformly smooth Banach space with the best smooth constant $K$. Then the following inequality holds:

$$
\|x+y\|^{2} \leq\|x\|^{2}+2\langle y, J x\rangle+2\|K y\|^{2}, \quad \forall x, y \in E .
$$

Lemma 2.4 (see [21]). Let $\left\{x_{n}\right\}$ and $\left\{y_{n}\right\}$ be bounded sequences in a Banach space $E$ and $\left\{\beta_{n}\right\}$ be a sequence in $[0,1]$ with

$$
0<\liminf _{n \rightarrow \infty} \beta_{n} \leq \limsup _{n \rightarrow \infty} \beta_{n}<1
$$

Suppose that $x_{n+1}=\left(1-\beta_{n}\right) y_{n}+\beta_{n} x_{n}$ for all $n \geq 0$ and

$$
\limsup _{n \rightarrow \infty}\left(\left\|y_{n+1}-y_{n}\right\|-\left\|x_{n+1}-x_{n}\right\|\right) \leq 0 .
$$

Then $\lim _{n \rightarrow \infty}\left\|y_{n}-x_{n}\right\|=0$.

Lemma 2.5 (see [22]). Assume that $\left\{a_{n}\right\}$ is a sequence of nonnegative real numbers such that

$$
a_{n+1} \leq\left(1-\alpha_{n}\right) a_{n}+\delta_{n}, \forall n \geq 0,
$$

where $\left\{\alpha_{n}\right\}$ is a sequence in $(0,1)$ and $\left\{\delta_{n}\right\}$ is a sequence in $\mathbb{R}$ such that

(1) $\sum_{n=1}^{\infty} \alpha_{n}=\infty$;

(2) $\lim \sup _{n \rightarrow \infty} \delta_{n} / \alpha_{n} \leq 0$ or $\sum_{n=1}^{\infty}\left|\delta_{n}\right|<\infty$.

Then $\lim _{n \rightarrow \infty} a_{n}=0$.

Lemma 2.6 (see [23]). Let $C$ be a closed convex subset of a strictly convex Banach space E. Let $T_{m}: C \rightarrow C$ be a nonexpansive mappings for each $1 \leq m \leq r$, where $r$ is some integer. Suppose that $\cap_{m=1}^{r} F\left(T_{m}\right)$ is nonempty. Let $\left\{\lambda_{n}\right\}$ be a sequence of positive numbers with $\sum_{m=1}^{r} \lambda_{n}=1$. Then the mapping $S: C \rightarrow C$ defined by

$$
S x=\sum_{m=1}^{r} \lambda_{m} T_{m} x, \quad \forall x \in C,
$$

is well defined, nonexpansive, and $F(S)=\cap_{m=1}^{r} F\left(T_{m}\right)$ holds.

Lemma 2.7 (see [24]). Let $C$ be a nonempty bounded closed convex subset of a uniformly convex Banach space $E$ and $T$ be nonexpansive mapping of $C$ into itself. If $\left\{x_{n}\right\}$ is a sequence in $C$ such that $x_{n} \rightarrow x$ weakly and $x_{n}-T x_{n} \rightarrow 0$ strongly, then $x$ is a fixed point of $T$.

Lemma 2.8 (see $[3,4]$ ). Let $C$ be a nonempty closed convex subset of a real 2-uniformly smooth Banach space E. Let a mapping $A: C \rightarrow E$ be $\lambda$-inverse-strongly accretive. Then one has

$$
\left\|\left(I-\rho_{2} A\right) x-\left(I-\rho_{2} A\right) y\right\|^{2} \leq\|x-y\|^{2}+2 \rho_{2}\left(\rho_{2} K^{2}-\lambda\right)\|A x-A y\|^{2} .
$$

If $\lambda \geq \rho_{2} K^{2}$, then $I-\rho_{2} A$ is nonexpansive. 
Proof. For any $x, y \in C$, it follows from Lemma 2.3 that

$$
\begin{aligned}
\left\|\left(I-\rho_{2} A\right) x-\left(I-\rho_{2} A\right) y\right\|^{2} & =\left\|(x-y)-\rho_{2}(A x-A y)\right\|^{2} \\
& \leq\|x-y\|^{2}-2 \rho_{2}\langle A x-A y, j(x-y)\rangle+2 \rho_{2}^{2} K^{2}\|A x-A y\|^{2} \\
& \leq\|x-y\|^{2}-2 \rho_{2} \lambda\|A x-A y\|^{2}+2 \rho_{2}^{2} K^{2}\|A x-A y\|^{2} \\
& =\|x-y\|^{2}+2 \rho_{2}\left(\rho_{2} K^{2}-\lambda\right)\|A x-A y\|^{2} .
\end{aligned}
$$

If $\lambda \geq \rho_{2} K^{2}$, then $I-\rho_{2} A$ is nonexpansive. This completes the proof.

Lemma 2.9. Let $C$ be a nonempty subset of a Banach space $E$. Let $A$ be a mapping of $C$ into $E, M$ be a maximal accretive operator on $E$ and $J_{M, \rho}=(I+\rho M)^{-1}$ be the resolvent of $M$ for any $\rho>0$. Then $F\left(J_{M, \rho}(I-\rho A)\right)=(A+M)^{-1}(0)$ for all $\rho>0$.

Proof. Let $\rho>0$ be fixed. Then we have

$$
\begin{aligned}
u \in F\left(J_{M, \rho}(I-\rho A)\right) u & \Longleftrightarrow u=J_{M, \rho}(I-\rho A) u=(I+\rho M)^{-1}(I-\rho A) u \\
& \Longleftrightarrow(I+\rho M) u \ni(I-\rho A) u \\
& \Longleftrightarrow \rho M u \ni-\rho A u \\
& \Longleftrightarrow M u \ni-A u \\
& \Longleftrightarrow(A+M) u \ni 0 \\
& \Longleftrightarrow u \in(A+M)^{-1}(0) .
\end{aligned}
$$

This completes the proof.

Lemma 2.10. Let $E$ be a Banach space. Then for all $x, y \in E$,

$$
\|x+y\|^{2} \leq\|x\|^{2}+2\langle y, J(x+y)\rangle
$$

\section{Main Results}

In this section, we prove strong convergence theorems for a $\lambda$-inverse-strongly accretive mapping $A: C \rightarrow E$ and a $\beta$-inverse-strongly accretive $B: C \rightarrow E$ in a real 2-uniformly smooth Banach space $E$.

In order to prove our main results, we need the following lemma.

Lemma 3.1. Let $C$ be a nonempty closed convex subset of a real 2-uniformly smooth Banach space $E$ with the best smooth constant $K$. Let $J_{M_{1}, \rho_{1}}, J_{M_{2}, \rho_{2}}$ be a resolvent operator associated with $M_{1}, M_{2}$, 
where $M_{1}, M_{2}: E \rightarrow 2^{E}$ is a multivalued maximal accretive mapping. Let the mappings $A, B: C \rightarrow$ $E$ be $\lambda$-inverse-strongly accretive and $\beta$-inverse-strongly accretive, respectively. Let $G: C \rightarrow C$ be a mapping defined by

$$
G x=\delta J_{M_{1}, \rho_{1}}\left(x-\rho_{1} B x\right)+(1-\delta) J_{M_{2}, \rho_{2}}\left(x-\rho_{2} A x\right), \quad \forall x \in C .
$$

If $\lambda \geq \rho_{2} K^{2}$ and $\beta \geq \rho_{1} K^{2}$, then $G$ is nonexpansive.

Proof. Since $J_{M_{1}, \rho_{1}}$ and $J_{M_{2}, \rho_{2}}$ are nonexpansive, for any $x, y \in C$, it follows from Lemma 2.8 that

$$
\begin{aligned}
\|G(x)-G(y)\|= & \| \delta J_{M_{1}, \rho_{1}}\left(x-\rho_{1} B x\right)+(1-\delta) J_{M_{2}, \rho_{2}}\left(x-\rho_{2} A x\right) \\
& -\delta J_{M_{1}, \rho_{1}}\left(y-\rho_{1} B y\right)-(1-\delta) J_{M_{2}, \rho_{2}}\left(y-\rho_{2} A y\right) \| \\
\leq & \delta\left\|J_{M_{1}, \rho_{1}}\left(x-\rho_{1} B x\right)-J_{M_{1}, \rho_{1}}\left(y-\rho_{1} B y\right)\right\| \\
& +(1-\delta)\left\|J_{M_{2}, \rho_{2}}\left(x-\rho_{2} A x\right)-J_{M_{2}, \rho_{2}}\left(y-\rho_{2} A y\right)\right\| \\
\leq & \delta\left\|J_{M_{1}, \rho_{1}}\left(I-\rho_{1} B\right) x-J_{M_{1}, \rho_{1}}\left(I-\rho_{1} B\right) y\right\| \\
& +(1-\delta)\left\|J_{M_{2}, \rho_{2}}\left(I-\rho_{2} A\right) x-J_{M_{2}, \rho_{2}}\left(I-\rho_{2} A\right) y\right\| \\
\leq & \|x-y\| .
\end{aligned}
$$

Therefore, $G$ is nonexpansive. This completes the proof.

Next, we state the main result of this work.

Theorem 3.2. Let $E$ be a uniformly convex and 2-uniformly smooth Banach space which admits a weakly sequentially continuous duality mapping and $C$ be a nonempty closed convex subset of E. Let $A, B: C \rightarrow E$ be $\lambda$-inverse-strongly accretive and $\beta$-inverse-strongly accretive, respectively, and $K$ be the best smooth constant. Let $f$ be a contraction of $E$ into itself with coefficient $\alpha \in[0,1)$. Suppose that $\Omega:=\left(A+M_{2}\right)^{-1}(0) \cap\left(B+M_{1}\right)^{-1}(0) \neq \emptyset$ and $G$ is a mapping defined by Lemma 3.1. Let $\rho_{1}, \rho_{2}$ be any positive real numbers such that $\rho_{1} \leq \beta / K^{2}$ and $\rho_{2} \leq \lambda / K^{2}$. For arbitrary $x_{0}=x \in C$, define the iterative sequence $\left\{x_{n}\right\}$ as follows:

$$
\begin{aligned}
x_{0} & =u \in C, \\
y_{n} & =\delta_{n} J_{M_{1}, \rho_{1}}\left(x_{n}-\rho_{1} B x_{n}\right)+\left(1-\delta_{n}\right) J_{M_{2}, \rho_{2}}\left(x_{n}-\rho_{2} A x_{n}\right), \\
x_{n+1} & =\alpha_{n} f\left(x_{n}\right)+\beta_{n} x_{n}+\gamma_{n} y_{n},
\end{aligned}
$$

where the sequences $\left\{\alpha_{n}\right\},\left\{\beta_{n}\right\}$, and $\left\{\gamma_{n}\right\}$ in $(0,1)$ satisfy the following conditions:

(C1) $\alpha_{n}+\beta_{n}+\gamma_{n}=1$;

(C2) $\lim _{n \rightarrow \infty} \alpha_{n}=0$ and $\sum_{n=0}^{\infty} \alpha_{n}=\infty$;

(C3) $0<\liminf _{n \rightarrow \infty} \beta_{n} \leq \lim \sup _{n \rightarrow \infty} \beta_{n}<1$;

(C4) $\lim _{n \rightarrow \infty} \delta_{n}=\delta \in(0,1)$. 
Then the sequence $\left\{x_{n}\right\}$ generated by (3.3) converges strongly to a point $q=Q_{\Omega} f(q)$, where $Q_{\Omega}$ is a sunny nonexpansive retraction on $\Omega$.

Proof. First, we prove that $J_{M_{1}, \rho_{1}}\left(I-\rho_{1} B\right)$ and $J_{M_{2}, \rho_{2}}\left(I-\rho_{2} A\right)$ are nonexpansive mappings. Consider the following:

$$
\begin{aligned}
\left\|J_{M_{1}, \rho_{1}}\left(I-\rho_{1} B\right) x-J_{M_{1}, \rho_{1}}\left(I-\rho_{1} B\right) y\right\|^{2} \leq & \left\|(x-y)-\rho_{1}(B x-B y)\right\|^{2} \\
\leq & \|x-y\|^{2}-2 \rho_{1}\langle B x-B y, J(x-y)\rangle \\
& +2 K^{2} \rho_{1}^{2}\|B x-B y\|^{2} \\
\leq & \|x-y\|^{2}-2 \rho_{1} \beta\|B x-B y\|^{2}+2 K^{2} \rho_{1}^{2}\|B x-B y\|^{2} \\
= & \|x-y\|^{2}+2 \rho_{1}\left(\rho_{1} K^{2}-\beta\right)\|B x-B y\|^{2} \\
\leq & \|x-y\|^{2} .
\end{aligned}
$$

Thus, it follows that $J_{M_{1}, \rho_{1}}\left(I-\rho_{1} B\right)$ is nonexpansive and so is $J_{M_{2}, \rho_{2}}\left(I-\rho_{2} A\right)$.

Step 1 . We show that $\left\{x_{n}\right\}$ is bounded. For any $p \in \Omega$, we have

$$
\begin{aligned}
\left\|y_{n}-p\right\| & =\left\|\delta_{n}\left[J_{M_{1}, \rho_{1}}\left(x_{\mathrm{n}}-\rho_{1} B x_{n}\right)-p\right]+\left(1-\delta_{n}\right)\left[J_{M_{2}, \rho_{2}}\left(x_{n}-\rho_{2} A x_{n}\right)-p\right]\right\| \\
& \leq \delta_{n}\left\|J_{M_{1}, \rho_{1}}\left(x_{n}-\rho_{1} B x_{n}\right)-p\right\|+\left(1-\delta_{n}\right)\left\|J_{M_{2}, \rho_{2}}\left(x_{n}-\rho_{2} A x_{n}\right)-p\right\| \\
& \leq \delta_{n}\left\|x_{n}-p\right\|+\left(1-\delta_{n}\right)\left\|x_{n}-p\right\| \\
& \leq\left\|x_{n}-p\right\| .
\end{aligned}
$$

It follows by induction that

$$
\begin{aligned}
\left\|x_{n+1}-p\right\| & =\left\|\alpha_{n} f\left(x_{n}\right)+\beta_{n} x_{n}+\gamma_{n} y_{n}-p\right\| \\
& \leq \alpha_{n}\left\|f\left(x_{n}\right)-p\right\|+\beta_{n}\left\|x_{n}-p\right\|+\gamma_{n}\left\|y_{n}-p\right\| \\
& \leq \alpha \alpha_{n}\left\|x_{n}-p\right\|+\alpha_{n}\|f(p)-p\|+\beta_{n}\left\|x_{n}-p\right\|+\gamma_{n}\left\|x_{n}-p\right\| \\
& \leq \alpha \alpha_{n}\left\|x_{n}-p\right\|+\alpha_{n}\|f(p)-p\|+\beta_{n}\left\|x_{n}-p\right\|+\gamma_{n}\left\|x_{n}-p\right\| \\
& =\left(1-\alpha_{n}+\alpha \alpha_{n}\right)\left\|x_{n}-p\right\|+\alpha_{n}\|f(p)-p\| \\
& =\left(1-\alpha_{n}(1-\alpha)\right)\left\|x_{n}-p\right\|+\alpha_{n}(1-\alpha) \frac{\|f(p)-p\|}{1-\alpha}
\end{aligned}
$$




$$
\begin{aligned}
& \leq \max \left\{\left\|x_{n}-p\right\|, \frac{\|f(p)-p\|}{1-\alpha}\right\} \\
& \ldots \\
& \leq \max \left\{\left\|x_{1}-p\right\|, \frac{\|f(p)-p\|}{1-\alpha}\right\} .
\end{aligned}
$$

Thus the sequence $\left\{x_{n}\right\}$ is bounded and so is $\left\{y_{n}\right\}$.

Step 2. We show that $\lim _{n \rightarrow \infty}\left\|x_{n+1}-x_{n}\right\|=0$. Let $u_{n}=J_{M_{1}, \rho_{1}}\left(I-\rho_{1} B\right) x_{n}$ and $v_{n}=J_{M_{2}, \rho_{2}}(I-$ $\left.\rho_{2} A\right) x_{n}$ for each $n \geq 0$. Then we have

$$
\begin{aligned}
y_{n+1}-y_{n} & =\left(\delta_{n+1} u_{n+1}+\left(1-\delta_{n+1}\right) v_{n+1}\right)-\left(\delta_{n} u_{n}+\left(1-\delta_{n}\right) v_{n}\right) \\
& =\delta_{n+1}\left(u_{n+1}-u_{n}\right)+\left(\delta_{n+1}-\delta_{n}\right)\left(u_{n}-v_{n}\right)+\left(1-\delta_{n+1}\right)\left(v_{n+1}-v_{n}\right),
\end{aligned}
$$

and so

$$
\begin{aligned}
\left\|y_{n+1}-y_{n}\right\| & \leq \delta_{n+1}\left\|u_{n+1}-u_{n}\right\|+\left|\delta_{n+1}-\delta_{n}\right|\left\|u_{n}-v_{n}\right\|+\left(1-\delta_{n+1}\right)\left\|v_{n+1}-v_{n}\right\| \\
& \leq \delta_{n+1}\left\|x_{n+1}-x_{n}\right\|+\left|\delta_{n+1}-\delta_{n}\right| M_{1}+\left(1-\delta_{n+1}\right)\left\|x_{n+1}-x_{n}\right\| \\
& =\left\|x_{n+1}-x_{n}\right\|+\left|\delta_{n+1}-\delta_{n}\right| M_{1}
\end{aligned}
$$

where $M_{1}$ ia an appropriate constant such that $M_{1} \geq \sup _{n \geq 0}\left\{\left\|u_{n}-v_{n}\right\|\right\}$.

Next, let $z_{n}=\left(x_{n+1}-\beta_{n} x_{n}\right) /\left(1-\beta_{n}\right)$ for all $n \geq 0$. Then we have $x_{n+1}=\left(1-\beta_{n}\right) z_{n}+\beta_{n} x_{n}$ for all $n \geq 0$. Now, we compute

$$
\begin{aligned}
z_{n+1}-z_{n} & =\frac{x_{n+2}-\beta_{n+1} x_{n+1}}{1-\beta_{n+1}}-\frac{x_{n+1}-\beta_{n} x_{n}}{1-\beta_{n}} \\
& =\frac{\alpha_{n+1} f\left(x_{n+1}\right)+\gamma_{n+1} y_{n+1}}{1-\beta_{n+1}}-\frac{\alpha_{n} f\left(x_{n}\right)+\gamma_{n} y_{n}}{1-\beta_{n}} \\
& =\frac{\alpha_{n+1} f\left(x_{n+1}\right)+\left(1-\alpha_{n+1}-\beta_{n+1}\right) y_{n+1}}{1-\beta_{n+1}}-\frac{\alpha_{n} f\left(x_{n}\right)+\left(1-\alpha_{n}-\beta_{n}\right) y_{n}}{1-\beta_{n}} \\
& =\frac{\alpha_{n+1} f\left(x_{n+1}\right)-\alpha_{n+1} y_{n+1}}{1-\beta_{n+1}}-\frac{\alpha_{n} f\left(x_{n}\right)-\alpha_{n} y_{n}}{1-\beta_{n}}+y_{n+1}-y_{n},
\end{aligned}
$$

and so

$$
\left\|z_{n+1}-z_{n}\right\| \leq \frac{\alpha_{n+1}}{1-\beta_{n+1}}\left\|f\left(x_{n+1}\right)-y_{n+1}\right\|+\frac{\alpha_{n}}{1-\beta_{n}}\left\|f\left(x_{n}\right)-y_{n}\right\|+\left\|y_{n+1}-y_{n}\right\| .
$$


Substituting (3.8) into (3.10), we get

$$
\begin{aligned}
\left\|z_{n+1}-z_{n}\right\| \leq & \frac{\alpha_{n+1}}{1-\beta_{n+1}}\left\|f\left(x_{n+1}\right)-y_{n+1}\right\|+\frac{\alpha_{n}}{1-\beta_{n}}\left\|f\left(x_{n}\right)-y_{n}\right\| \\
& +\left\|x_{n+1}-x_{n}\right\|+\left|\delta_{n+1}-\delta_{n}\right| M_{1}
\end{aligned}
$$

that is,

$$
\left\|z_{n+1}-z_{n}\right\|-\left\|x_{n+1}-x_{n}\right\| \leq \frac{\alpha_{n+1}}{1-\beta_{n+1}}\left\|f\left(x_{n+1}\right)-y_{n+1}\right\|+\frac{\alpha_{n}}{1-\beta_{n}}\left\|f\left(x_{n}\right)-y_{n}\right\|+\left|\delta_{n+1}-\delta_{n}\right| M_{1}
$$

From the conditions (C2) and (C3), it follows that

$$
\limsup _{n \rightarrow \infty}\left(\left\|z_{n+1}-z_{n}\right\|-\left\|x_{n+1}-x_{n}\right\|\right) \leq 0
$$

Thus, from Lemma 2.4, it follows that

$$
\lim _{n \rightarrow \infty}\left\|z_{n}-x_{n}\right\|=0
$$

From the definition of $x_{n+1}$ in this step, we observe that $x_{n+1}-x_{n}=\left(1-\beta_{n}\right)\left(z_{n}-x_{n}\right)$. Then we have

$$
\begin{aligned}
\lim _{n \rightarrow \infty}\left\|x_{n+1}-x_{n}\right\| & =\lim _{n \rightarrow \infty}\left\|\left(1-\beta_{n}\right)\left(z_{n}-x_{n}\right)\right\| \\
& =0 .
\end{aligned}
$$

Step 3. We show that $\lim \sup _{n \rightarrow \infty}\left\langle(f-I) q, J\left(x_{n}-q\right)\right\rangle \leq 0$, where $q=Q_{\Omega} f(q)$. Define a mapping $G: C \rightarrow C$ by Lemma 3.1 Then, it follows that $G$ is a nonexpansive mapping such that

$$
F(G)=F\left(J_{M_{1}, \rho_{1}}\left(I-\rho_{1} B\right)\right) \cap F\left(J_{M_{2}, \rho_{2}}\left(I-\rho_{2} A\right)\right)=(B+M)^{-1}(0) \cap(A+M)^{-1}(0)=\Omega .
$$

Consider the following:

$$
\begin{aligned}
y_{n}-G x_{n} & =\delta_{n} v_{n}+\left(1-\delta_{n}\right) u_{n}-\left(\delta v_{n}+(1-\delta) u_{n}\right) \\
& =\left(\delta_{n}-\delta\right)\left(v_{n}-u_{n}\right) .
\end{aligned}
$$

From the condition (C4), we have

$$
\lim _{n \rightarrow \infty}\left\|y_{n}-G x_{n}\right\|=0
$$


Next, we consider

$$
\begin{aligned}
\left\|x_{n}-G x_{n}\right\| & =\left\|x_{n}-x_{n+1}+x_{n+1}-y_{n}+y_{n}-G x_{n}\right\| \\
& \leq\left\|x_{n}-x_{n+1}\right\|+\left\|x_{n+1}-y_{n}\right\|+\left\|y_{n}-G x_{n}\right\| \\
& \leq\left\|x_{n}-x_{n+1}\right\|+\alpha_{n}\left\|f\left(x_{n}\right)-y_{n}\right\|+\beta_{n}\left\|x_{n}-y_{n}\right\|+\left\|y_{n}-G x_{n}\right\| \\
& \leq\left\|x_{n}-x_{n+1}\right\|+\alpha_{n}\left\|f\left(x_{n}\right)-y_{n}\right\|+\beta_{n}\left\|x_{n}-G x_{n}\right\|+\beta_{n}\left\|G x_{n}-y_{n}\right\|+\left\|y_{n}-G x_{n}\right\| .
\end{aligned}
$$

Therefore, we have

$$
\left(1-\beta_{n}\right)\left\|x_{n}-G x_{n}\right\| \leq\left\|x_{n}-x_{n+1}\right\|+\alpha_{n}\left\|f\left(x_{n}\right)-y_{n}\right\|+\left(\beta_{n}+1\right)\left\|G x_{n}-y_{n}\right\| .
$$

From the conditions (C2), (C3), (3.15), (3.18), and the inequality above, we obtain

$$
\lim _{n \rightarrow \infty}\left\|x_{n}-G x_{n}\right\|=0
$$

Thus, since $Q_{\Omega} f(q)$ is a contraction, there exists a unique fixed point. We denote that $q$ is the unique fixed point to the mapping $Q_{\Omega} f(q)$ which means that $q=Q_{\Omega} f(q)$.

Since $\left\{x_{n}\right\}$ is bounded, there exists a subsequence $\left\{x_{n_{i}}\right\}$ of $\left\{x_{n}\right\}$ such that $x_{n_{i}} \rightarrow p$, it follows from (3.21) that

$$
\lim _{n \rightarrow \infty}\left\|x_{n_{i}}-G x_{n_{i}}\right\|=0
$$

Since $G$ is nonexpansive, it follows from Lemma 2.7 that $p=G p$ we obtain that $p \in F(G)$. By (3.22), we have $p \in \Omega$.

Furthermore, with the reason that $\left\{x_{n}\right\}$ is bounded, we can choose the sequence $\left\{x_{n_{i}}\right\}$ of $\left\{x_{n}\right\}$ which $\left\{x_{n_{i}}\right\} \rightarrow p$ such that

$$
\limsup _{n \rightarrow \infty}\left\langle(f-I) q, J\left(x_{n}-q\right)\right\rangle=\lim _{i \rightarrow \infty}\left\langle(f-I) q, J\left(x_{n_{i}}-q\right)\right\rangle
$$

Now, from (3.23) and Proposition 2.1(3) and the weakly sequential continuity of the duality mapping $J$, we have

$$
\begin{aligned}
\limsup _{n \rightarrow \infty}\left\langle(f-I) q, J\left(x_{n}-q\right)\right\rangle & =\lim _{i \rightarrow \infty}\left\langle(f-I) q, J\left(x_{n_{i}}-q\right)\right\rangle \\
& =\langle(f-I) q, J(p-q)\rangle \leq 0 .
\end{aligned}
$$

From (3.15), it follows that

$$
\limsup _{n \rightarrow \infty}\left\langle(f-I) q, J\left(x_{n+1}-q\right)\right\rangle \leq 0
$$


Step 4 . We show that $\left\{x_{n}\right\}$ converges strongly to a point $q=Q_{\Omega} f(q)$. In fact, observe that

$$
\begin{aligned}
\left\|x_{n+1}-q\right\|^{2}= & \left\langle x_{n+1}-q, J\left(x_{n+1}-q\right)\right\rangle \\
= & \left\langle\alpha_{n}\left(f\left(x_{n}\right)-q\right)+\beta_{n}\left(x_{n}-q\right)+\gamma_{n}\left(y_{n}-q\right), J\left(x_{n+1}-q\right)\right\rangle \\
\leq & \alpha \alpha_{n}\left\langle x_{n}-q, J\left(x_{n+1}-q\right)\right\rangle+\alpha_{n}\left\langle f(q)-q, J\left(x_{n+1}-q\right)\right\rangle \\
& +\beta_{n}\left\langle x_{n}-q, J\left(x_{n+1}-q\right)\right\rangle+\gamma_{n}\left\langle y_{n}-q, J\left(x_{n+1}-q\right)\right\rangle \\
\leq & \alpha \alpha_{n}\left\|x_{n}-q\right\|\left\|x_{n+1}-q\right\|+\alpha_{n}\left\langle f(q)-q, J\left(x_{n+1}-q\right)\right\rangle \\
& +\beta_{n}\left\|x_{n}-q\right\|\left\|x_{n+1}-q\right\|+\gamma_{n}\left\|y_{n}-q\right\|\left\|x_{n+1}-q\right\| \\
\leq & \left(1-\alpha_{n}(1-\alpha)\right)\left\|x_{n}-q\right\|\left\|x_{n+1}-q\right\|+\alpha_{n}\left\langle f(q)-q, J\left(x_{n+1}-q\right)\right\rangle \\
\leq & \frac{1-\alpha_{n}(1-\alpha)}{2}\left(\left\|x_{n}-q\right\|^{2}+\left\|x_{n+1}-q\right\|^{2}\right)+\alpha_{n}\left\langle f(q)-q, J\left(x_{n+1}-q\right)\right\rangle \\
\leq & \frac{1-\alpha_{n}(1-\alpha)}{2}\left\|x_{n}-q\right\|^{2}+\frac{1}{2}\left\|x_{n+1}-q\right\|^{2}+\alpha_{n}\left\langle f(q)-q, J\left(x_{n+1}-q\right)\right\rangle .
\end{aligned}
$$

Thus it follows that

$$
\left\|x_{n+1}-q\right\|^{2} \leq\left(1-\alpha_{n}(1-\alpha)\right)\left\|x_{n}-q\right\|^{2}+2 \alpha_{n}\left\langle f(q)-q, J\left(x_{n+1}-q\right)\right\rangle .
$$

Therefore, from Condition (C2), (3.25), and Lemma 2.5, we get $\left\|x_{n}-q\right\| \rightarrow 0$ as $n \rightarrow \infty$. This completes the proof.

Corollary 3.3. Let E be a uniformly convex and 2-uniformly smooth Banach space which admits a weakly sequentially continuous duality mapping and let $C$ be a nonempty closed convex subset of $E$. Let $A, B: C \rightarrow E$ be $\lambda$-inverse-strongly accretive and $\beta$-inverse-strongly accretive, respectively, and $K$ be the best smooth constant. Suppose that $\Omega:=\left(A+M_{2}\right)^{-1}(0) \cap\left(B+M_{1}\right)^{-1}(0) \neq \emptyset$, where $G$ is a mapping defined by Lemma 3.1. Let $\rho_{1}, \rho_{2}$ be any positive real numbers such that $\rho_{1} \leq \beta / K^{2}$ and $\rho_{2} \leq \lambda / K^{2}$. For arbitrary $x_{0}=x \in C$, define the iterative sequence $\left\{x_{n}\right\}$ by

$$
\begin{aligned}
x_{0} & =u \in C, \\
y_{n} & =\delta_{n} J_{M_{1}, \rho_{1}}\left(x_{n}-\rho_{1} B x_{n}\right)+\left(1-\delta_{n}\right) J_{M_{2}, \rho_{2}}\left(x_{n}-\rho_{2} A x_{n}\right), \\
x_{n+1} & =\alpha_{n} u+\beta_{n} x_{n}+\gamma_{n} y_{n}, \quad \forall n \geq 0,
\end{aligned}
$$

where the sequences $\left\{\alpha_{n}\right\},\left\{\beta_{n}\right\}$, and $\left\{\gamma_{n}\right\}$ in $(0,1)$ satisfy the following conditions:

(C1) $\alpha_{n}+\beta_{n}+\gamma_{n}=1$;

(C2) $\lim _{n \rightarrow \infty} \alpha_{n}=0$ and $\sum_{n=0}^{\infty} \alpha_{n}=\infty$;

(C3) $0<\liminf _{n \rightarrow \infty} \beta_{n} \leq \lim \sup _{n \rightarrow \infty} \beta_{n}<1$;

(C4) $\lim _{n \rightarrow \infty} \delta_{n}=\delta \in(0,1)$.

Then the sequence $\left\{x_{n}\right\}$ generated by (3.28) converges strongly to a point $q=Q_{\Omega} f(q)$, where $Q_{\Omega}$ is a sunny nonexpansive retraction on $\Omega$. 
Proof. Take $f\left(x_{n}\right)=u$ for all $n \geq 1$ for any fixed $u \in C$ in (3.3). Then, by Theorem 3.2, we can conclude the desired conclusion easily.

\section{Applications}

\subsection{Application to Convex Feasibility Problems}

In this part, we consider the following convex feasibility problem (CFP): find $x \in \cap_{j=1}^{N} C_{j}$, where $j \in\{1,2, \ldots, N\}$ and $C_{j}$ denotes the set of zeros of a maximal accretive operator.

The following result can be obtained from Theorem 3.2.

Theorem 4.1. Let E be a uniformly convex and 2-uniformly smooth Banach space which admits a weakly sequentially continuous duality mapping and let $C$ be a nonempty closed convex subset of $E$. Let $\left\{A_{i}\right\}_{i}=1^{N}: C \rightarrow E$ be an $\lambda_{i}$-inverse-strongly accretive and $K$ be the best smooth constant. Let $f$ be a contraction of $E$ into itself with coefficient $\alpha \in[0,1)$. Suppose that $\Omega:=\bigcap_{i=1}^{N}\left(A_{i}+M_{i}\right)^{-1}(0) \neq \emptyset$, where $G$ is a mapping defined by Lemma 3.1. Let $\rho_{i}$ be any positive real numbers such that $\rho_{i} \leq \lambda_{i} / K^{2}$, $i=1,2,3, \ldots, N$. For arbitrary $x_{0}=x \in C$, define the iterative sequence $\left\{x_{n}\right\}$ by

$$
\begin{aligned}
x_{0} & =u \in C, \\
y_{n} & =\sum_{i=1}^{N} \delta_{i, n} J_{M_{i}, \rho_{i}}\left(x_{n}-\rho_{i} A_{i} x_{n}\right), \\
x_{n+1} & =\alpha_{n} f\left(x_{n}\right)+\beta_{n} x_{n}+\gamma_{n} y_{n}, \quad \forall n \geq 0,
\end{aligned}
$$

where the sequences $\left\{\alpha_{n}\right\},\left\{\beta_{n}\right\}$, and $\left\{\gamma_{n}\right\}$ in $(0,1)$ satisfy the following conditions:

$$
\begin{aligned}
& \text { (C1) } \alpha_{n}+\beta_{n}+\gamma_{n}=1 \text { and } \sum_{i=1}^{N} \delta_{i, n}=1 \text {; } \\
& \text { (C2) } \lim _{n \rightarrow \infty} \alpha_{n}=0 \text { and } \sum_{n=0}^{\infty} \alpha_{n}=\infty \text {; } \\
& \text { (C3) } 0<\liminf _{n \rightarrow \infty} \beta_{n} \leq \limsup \sup _{n \rightarrow \infty} \beta_{n}<1 \text {; } \\
& \text { (C4) } \lim _{n \rightarrow \infty} \delta_{i, n}=\delta \in(0,1) \text {. }
\end{aligned}
$$

Then the sequence $\left\{x_{n}\right\}$ generated by (4.1) converges strongly to a point $q=Q_{\Omega}$, where $Q_{\Omega}$ is a sunny nonexpansive retraction on $\Omega$.

\subsection{Application to Hilbert Spaces}

Assume that $H$ is a real Hilbert space with inner product $\langle\cdot, \cdot\rangle$ and norm $\|\cdot\|$. Let $A: H \rightarrow H$ be a single-valued nonlinear mapping and $M: H \rightarrow 2^{H}$ be a multivalued mapping. The problem of finding $u \in H$ such that

$$
\theta \in A(u)+M(u)
$$

is called the quasivariational inclusion problem, and we denote the set of solutions of the above variational inclusion by $\mathrm{VI}(H, A, M)$. 
If $M=\partial \delta_{C}$, where $C$ is a nonempty closed convex subset of $H$ and $\delta_{C}: H \rightarrow[0, \infty]$ is the indicator function of $C$, that is,

$$
\delta_{C}(x)= \begin{cases}0, & x \in C, \\ +\infty, & x \notin C .\end{cases}
$$

Then the variational inclusion problem (4.2) is equivalent to the problem of finding $u \in C$ such that

$$
\langle A(u), v-u\rangle \geq 0, \quad \forall v \in C,
$$

which is the well-known Hartman-Stampacchia variational inequality problem [25].

Theorem 4.2. Let $C$ be a closed convex subset of a real Hilbert space $H$. Let $A, B: C \rightarrow H$ be $\lambda$-inverse-strongly monotone and $\beta$-inverse-strongly monotone, respectively. Let $f$ be a contraction of $E$ into itself with coefficient $\alpha \in[0,1)$. Suppose that $\Omega:=\operatorname{VI}(C, A) \cap \operatorname{VI}(C, B) \neq \emptyset$, where $\operatorname{VI}(C, A)$ and $\operatorname{VI}(C, B)$ are the sets of solutions of variational inequality (4.4). For arbitrary $x_{0}=x \in C$, define the iterative sequence $\left\{x_{n}\right\}$ by

$$
\begin{aligned}
x_{0} & =u \in C, \\
y_{n} & =\delta_{n} P_{C}\left(x_{n}-\rho_{1} B x_{n}\right)+\left(1-\delta_{n}\right) P_{C}\left(x_{n}-\rho_{2} A x_{n}\right), \\
x_{n+1} & =\alpha_{n} f\left(x_{n}\right)+\beta_{n} x_{n}+\gamma_{n} y_{n}, \quad \forall n \geq 0,
\end{aligned}
$$

where the sequences $\left\{\alpha_{n}\right\},\left\{\beta_{n}\right\}$, and $\left\{\gamma_{n}\right\}$ in $(0,1)$ satisfy the following conditions:

(C1) $\alpha_{n}+\beta_{n}+\gamma_{n}=1$;

(C2) $\lim _{n \rightarrow \infty} \alpha_{n}=0$ and $\sum_{n=0}^{\infty} \alpha_{n}=\infty$;

(C3) $0<\liminf _{n \rightarrow \infty} \beta_{n} \leq \lim \sup _{n \rightarrow \infty} \beta_{n}<1$;

(C4) $\lim _{n \rightarrow \infty} \delta_{n}=\delta \in(0,1)$.

Then the sequence $\left\{x_{n}\right\}$ generated by (4.5) converges strongly to a point $P_{\Omega} x_{0}$.

Proof. Take $M=\partial \delta_{C}: H \rightarrow 2^{H}$, where $\delta_{C}: H \rightarrow[0, \infty]$ is the indicator function of $C$. Let $J(M, \rho)=I$. Then we get

$$
\begin{aligned}
& P_{C}\left(x_{n}-\rho_{1} A x_{n}\right)=J_{M, \rho_{1}} P_{C}\left(x_{n}-\rho_{1} A x_{n}\right), \\
& P_{C}\left(x_{n}-\rho_{2} B x_{n}\right)=J_{M, \rho_{2}} P_{C}\left(x_{n}-\rho_{2} B x_{n}\right) .
\end{aligned}
$$

Thus the conclusion can be obtained from Theorem 3.2 immediately.

\section{Acknowledgments}

This paper was supported by the Higher Education Research Promotion and National Research University Project of Thailand, Office of the Higher Education Commission (under 
the project NRU-CSEC no. 54000267) for financial support during the preparation of this paper. Furthermore, the first author would like to thank the Office of the Higher Education Commission, Thailand, for the financial support of the Ph.D. program at KMUTT. This research was partially finished at Department of Mathematics Education, Gyeongsang National University, Republic of Korea. Also, the second author was supported by the Basic Science Research Program through the National Research Foundation of Korea (NRF) funded by the Ministry of Education, Science, and Technology (Grant no. 2011-0021821).

\section{References}

[1] W. Takahashi, Nonlinear Functional Analysis: Fixed Point Theory and its Applications, Yokohama Publishers, Yokohama, Japan, 2000.

[2] W. Takahashi, "Viscosity approximation methods for resolvents of accretive operators in Banach spaces," Journal of Fixed Point Theory and Applications, vol. 1, no. 1, pp. 135-147, 2007.

[3] Y. Yao, M. Aslam Noor, K. Inayat Noor, Y. C. Liou, and H. Yaqoob, "Modified extragradient methods for a system of variational inequalities in Banach spaces," Acta Applicandae Mathematicae, vol. 110, no. 3, pp. 1211-1224, 2010.

[4] K. Aoyama, H. Iiduka, and W. Takahashi, "Weak convergence of an iterative sequence for accretive operators in Banach spaces," Fixed Point Theory and Applications, vol. 2006, Article ID 35390, 13 pages, 2006.

[5] H. K. Xu, "Inequalities in Banach spaces with applications," Nonlinear Analysis, vol. 16, no. 12, pp. 1127-1138, 1991.

[6] R. P. Agarwal, H. Zhou, Y. J. Cho, and S. M. Kang, "Zeros and mapping theorems for perturbations of $m$-accretive operators in Banach spaces," Computers $\mathcal{E}$ Mathematics with Applications, vol. 49, no. 1, pp. 147-155, 2005.

[7] Y. J. Cho, S. M. Kang, and H. Zhou, "Approximate proximal point algorithms for finding zeroes of maximal monotone operators in Hilbert spaces," Journal of Inequalities and Applications, vol. 2008, Article ID 598191, 10 pages, 2008.

[8] P. Cholamjiak, Y. J. Cho, and S. Suantai, "Composite iterative schemes for maximal monotone operators in reflexive Banach spaces," Fixed Point Theory and Applications, vol. 2011, article 7, 2011.

[9] S. Kamimura and W. Takahashi, "Approximating solutions of maximal monotone operators in Hilbert spaces," Journal of Approximation Theory, vol. 106, no. 2, pp. 226-240, 2000.

[10] S. Kamimura and W. Takahashi, "Weak and strong convergence of solutions to accretive operator inclusions and applications," Set-Valued Analysis, vol. 8, no. 4, pp. 361-374, 2000.

[11] X. Qin, Y. J. Cho, and S. M. Kang, "Approximating zeros of monotone operators by proximal point algorithms," Journal of Global Optimization, vol. 46, no. 1, pp. 75-87, 2010.

[12] Y. Song, J. I. Kang, and Y. J. Cho, "On iterations methods for zeros of accretive operators in Banach spaces," Applied Mathematics and Computation, vol. 216, no. 3, pp. 1007-1017, 2010.

[13] L. Wei and Y. J. Cho, "Iterative schemes for zero points of maximal monotone operators and fixed points of nonexpansive mappings and their applications," Fixed Point Theory and Applications, vol. 2008, Article ID 168468, 12 pages, 2008.

[14] H. Iiduka, W. Takahashi, and M. Toyoda, "Approximation of solutions of variational inequalities for monotone mappings," Panamerican Mathematical Journal, vol. 14, no. 2, pp. 49-61, 2004.

[15] P. Katchang and P. Kumam, "Convergence of iterative algorithm for finding common solution of fixed points and general system of variational inequalities for two accretive operators," Thai Journal of Mathematics, vol. 9, no. 2, pp. 343-360, 2011.

[16] N. Petrot, R. Wangkeeree, and P. Kumam, "A viscosity approximation method of common solutions for quasi variational inclusion and fixed point problems," Fixed Point Theory, vol. 12, no. 1, pp. 165178, 2011.

[17] X. Qin, S. Y. Cho, and Y. J. Cho, "Approximating common solutions of variational inequalities by iterative algorithms with applications," Glasnik Matematički, vol. 46, no. 1, pp. 233-247, 2011.

[18] W. Takahashi, Convex Analysis and Approximation Fixed Points, vol. 2 of Mathematical Analysis Series, Yokohama Publishers, Yokohama, Japan, 2000.

[19] S. Reich, "Asymptotic behavior of contractions in Banach spaces," Journal of Mathematical Analysis and Applications, vol. 44, pp. 57-70, 1973. 
[20] S. Kitahara and W. Takahashi, "Image recovery by convex combinations of sunny nonexpansive retractions," Topological Methods in Nonlinear Analysis, vol. 2, no. 2, pp. 333-342, 1993.

[21] T. Suzuki, "Strong convergence of Krasnoselskii and Mann's type sequences for one-parameter nonexpansive semigroups without Bochner integrals," Journal of Mathematical Analysis and Applications, vol. 305, no. 1, pp. 227-239, 2005.

[22] H. K. Xu, "Viscosity approximation methods for nonexpansive mappings," Journal of Mathematical Analysis and Applications, vol. 298, no. 1, pp. 279-291, 2004.

[23] R. E. Bruck, "Properties of fixed-point sets of nonexpansive mappings in Banach spaces," Transactions of the American Mathematical Society, vol. 179, pp. 251-262, 1973.

[24] F. E. Browder, "Nonlinear operators and nonlinear equations of evolution in Banach spaces," in Proceedings of the Symposia in Pure Mathematics, vol. 18, pp. 78-81, 1976.

[25] P. Hartman and G. Stampacchia, "On some non-linear elliptic differential-functional equations," Acta Mathematica, vol. 115, pp. 271-310, 1966. 


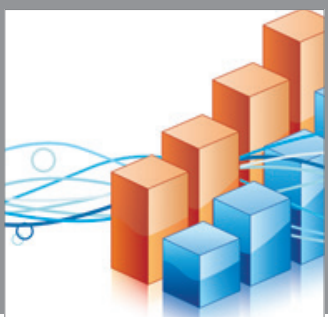

Advances in

Operations Research

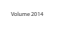

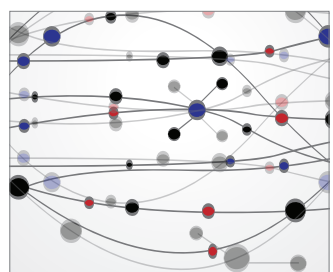

\section{The Scientific} World Journal
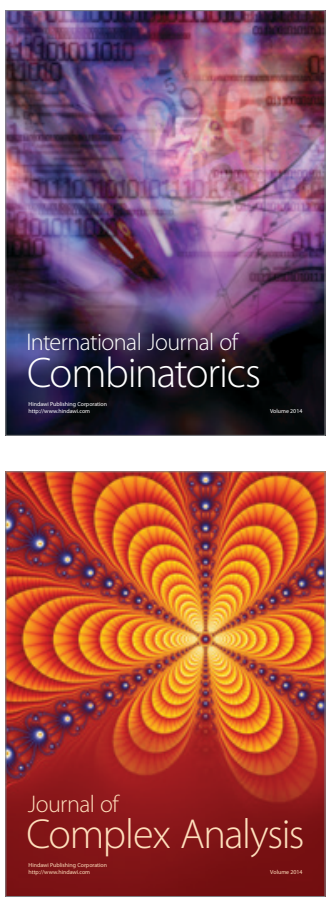

International Journal of

Mathematics and

Mathematical

Sciences
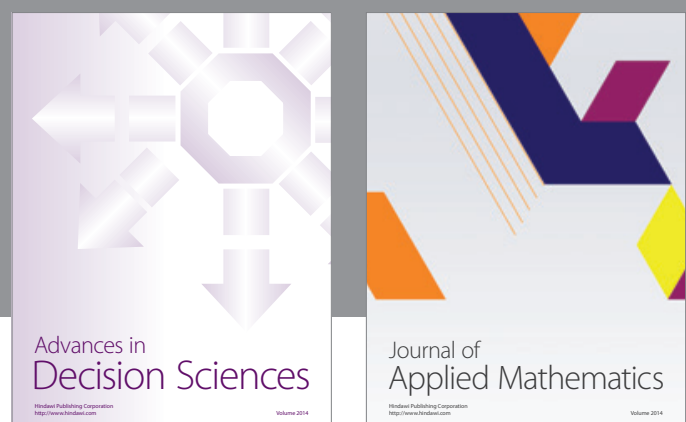

Journal of

Applied Mathematics
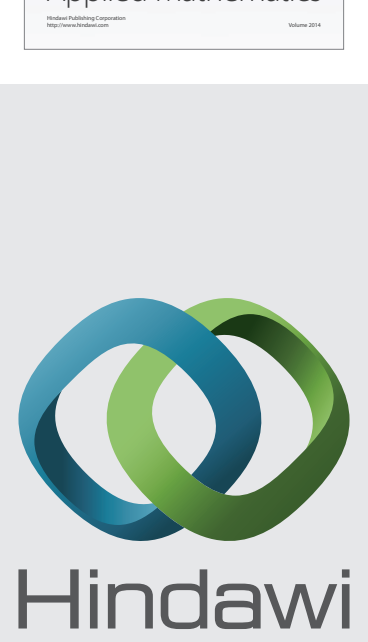

Submit your manuscripts at http://www.hindawi.com
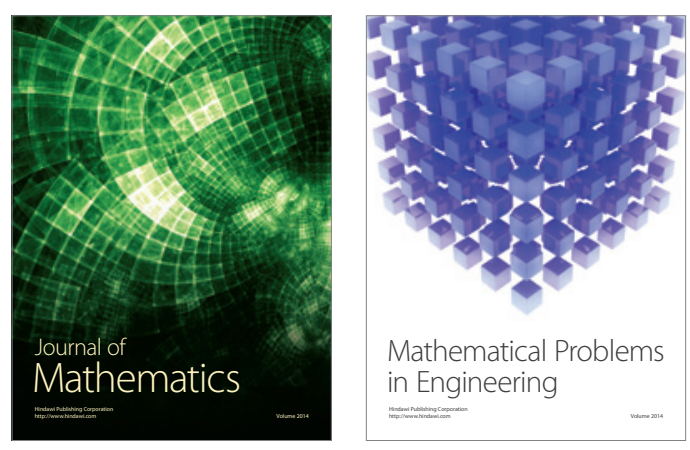

Mathematical Problems in Engineering
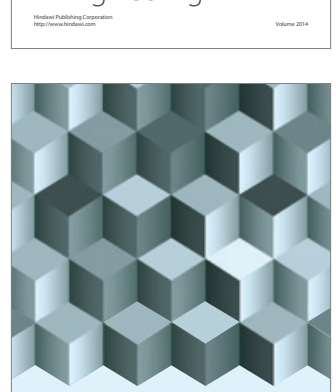

Journal of

Function Spaces
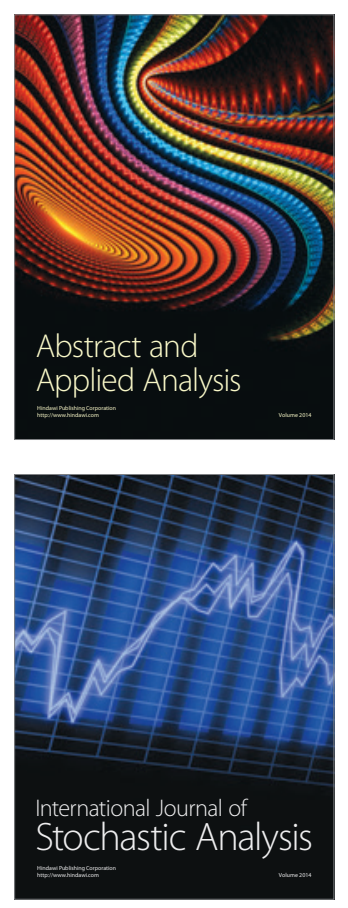

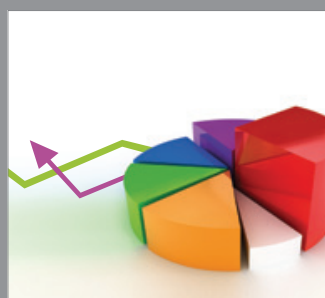

ournal of

Probability and Statistics

Promensencen
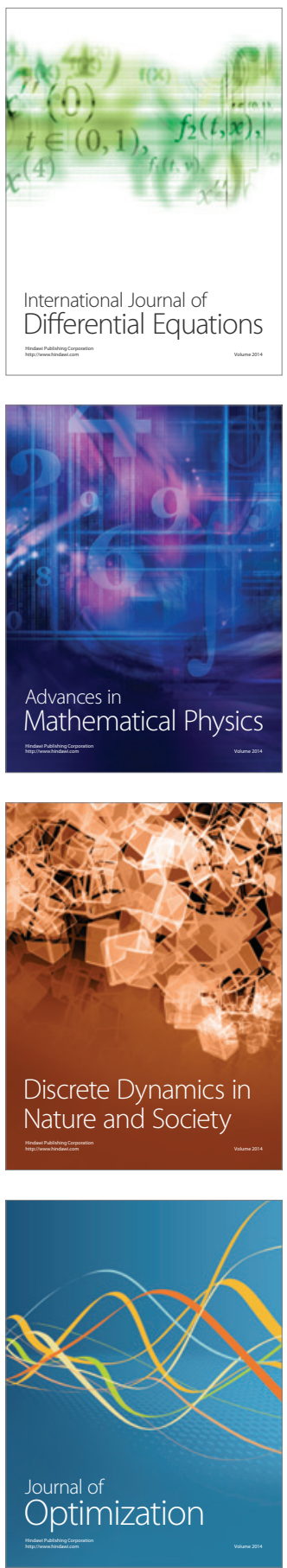